\title{
Development of an isolated retinal macroaneurysm of the cilioretinal artery
}

\author{
GIUSEPPE GIUFFRE, FRANCESCO PAOLO MONTALTO, \\ AND GIUSEPPE AMODEI \\ From the Istituto di Clinica Oculistica dell'Università, Palermo, Italy
}

SUMMARY A case of isolated retinal macroaneurysm showed interesting features. First, it involved a cilioretinal artery, which is a rare site; secondly, its development was preceded by vessel changes characterised by hyalinisation and permeability of the arterial wall.

Isolated retinal macroaneurysms are acquired dilatations of the major branches of the central retinal artery. ${ }^{1}$ They are found in subjects with vasculopathies, especially arterial hypertension and arteriosclerosis. ${ }^{1-5}$ Frequently macroaneurysms produce complications such as oedema, retinal, subretinal and vitreous haemorrhages, and serous detachment of the retina, generally in the posterior pole. ${ }^{2356} \mathrm{The}$ pathogenesis of macroaneurysms is not well known and the vessel abnormalities that precede their development have rarely been demonstrated.?

We have followed the development of a macroaneurysm located in a cilioretinal artery, a very rare occurrence, and have observed some interesting changes in the arterial wall heralding the formation of the macroaneurysm. The recognition of these changes could be important in the selection of patients at risk of developing macroaneurysms.

\section{Case report}

A 70-year-old woman complaining of a bilateral decrease in visual acuity was referred on 27 August 1983 to our service of fluorescein angiography with a diagnosis of hypertensive retinopathy. She had suffered from arterial hypertension for many years; her therapy included hydrochlorothiazide, alphamethyldopa, and platelet anti-aggregating drugs. At the time of the visit her blood pressure was 180/100 $\mathrm{mmHg}$. A slight increase in plasma lipids (cholesterol $8.5 \mathrm{mmol} / \mathrm{l}$; triglicerides $3.2 \mathrm{mmol} / \mathrm{l}$ ) was found.

Correspondence to $\mathrm{Dr}$ Giuseppe Giuffrè, Istituto di Clinica Oculistica, Via L Giuffrè 13, 90127, Palermo, Italy.
The corrected visual acuity was $20 / 40$ in the right eye and 20/200 in the left eye. There was an early nuclear cataract in the right eye and a more advanced nuclear and posterior subcapsular cataract in the left eye.

Examination of the fundus of the right eye showed narrowing of the retinal arteries and compression at arteriovenous crossings. Some tracts of the nasal and superotemporal arteries were hyalinised, and along their course there were some round haemorrhages. A macular cilioretinal artery was hyalinised and surrounded by some hard exudates in its supramacular tract (Fig. 1). A chorioretinal scar was seen in the superotemporal fundus, and some small haemorrhages and hard exudates were observed in the macula. The fundus of the left eye showed arterial narrowing, abnormal arteriovenous crossings, haemorrhages, and hard exudates in the posterior pole. There was neither hyalinisation nor occlusion of retinal arteries.

Fluorescein angiography in the right eye revealed occlusion of some branches of the nasal and superotemporal arteries. The cilioretinal artery was normally filled and in its hyalinised tract showed an irregular sausage-like dilatation (Fig. 2). There was no leakage.

On 20 April 1984 the corrected visual acuity was $20 / 60$ in the right eye and 20/200 in the left eye.

Occlusion of an inferotemporal branch of the central retinal vein occurred. Ischaemia and slight oedema of the macula were observed. The lesion of the cilioretinal artery was unchanged. The patient refused argon laser treatment of the affected area. 


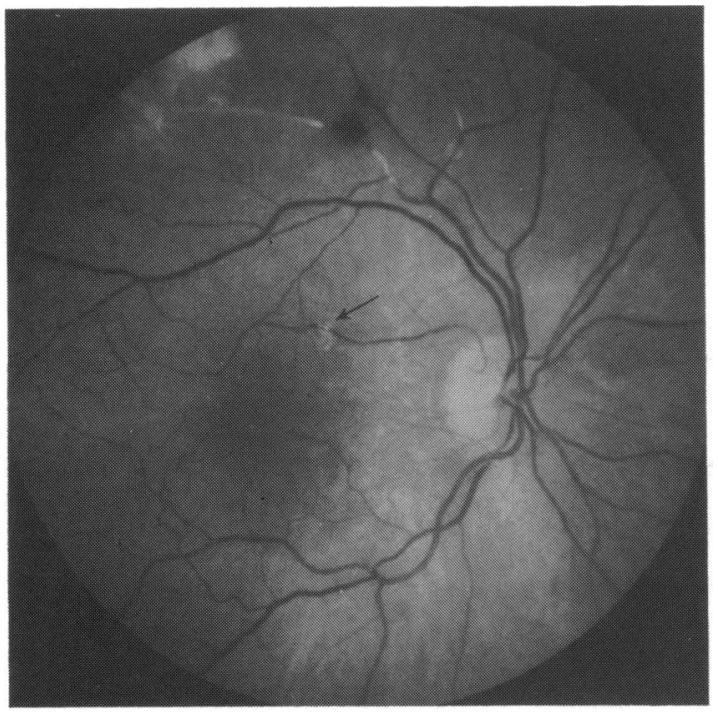

Fig. 1 A tract of the cilioretinal artery shows hyalinisation of the walls (arrow). Below it are some hard exudates. The superotemporal branch of the central retinal artery is partially hyalinised.

At the next examination, on 10 April 1986, the patient's blood pressure was $170 / 100 \mathrm{mmHg}$ and normal serum lipids. The visual acuity was the same as that found previously.

Along the cilioretinal artery, in the macula, there was a round, whitish ectasia, partly covered by a

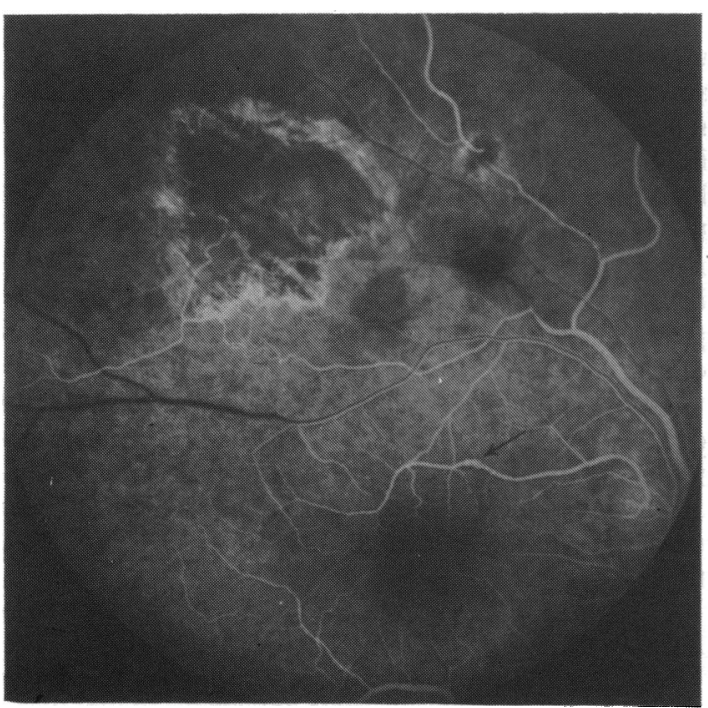

Fig. 2 Fluorescein angiography demonstrates that the hyalinised tract of the cilioretinal artery is slightly dilated (arrow). The superotemporal artery incompletely fills owing to occlusion.

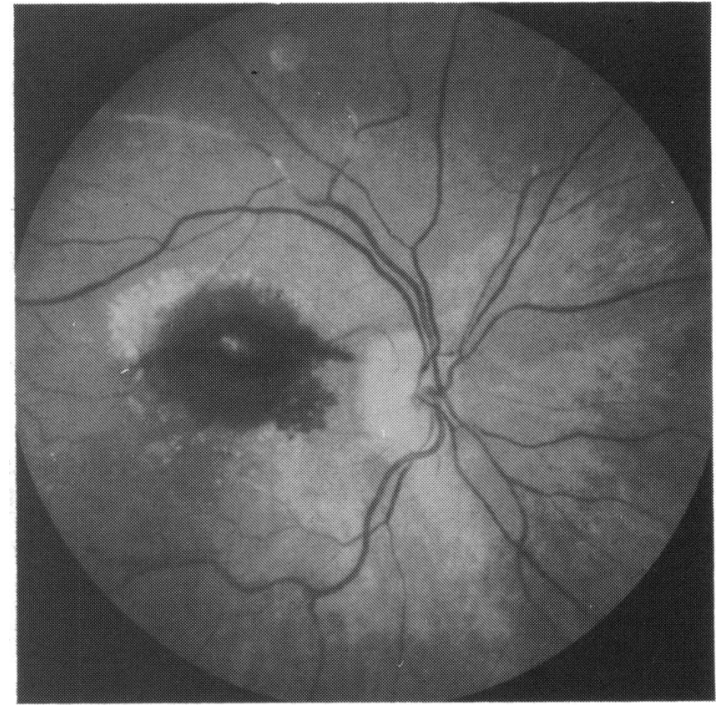

Fig. 3 The cilioretinal artery shows a macroaneurysm covered by a preretinal hemorrhage. In the same area a ring of hard exudates and a subretinal haemorrhage are seen.

preretinal haemorrhage (Fig. 3). In the same area of the fundus a subretinal haemorrhage and a ring of hard exudates were seen.

Fluorescein angiography showed normal filling of the cilioretinal artery. The aneurysmal dilatation filled at the same time as the artery and showed leakage (Fig. 4). The patient again refused photocoagulation.

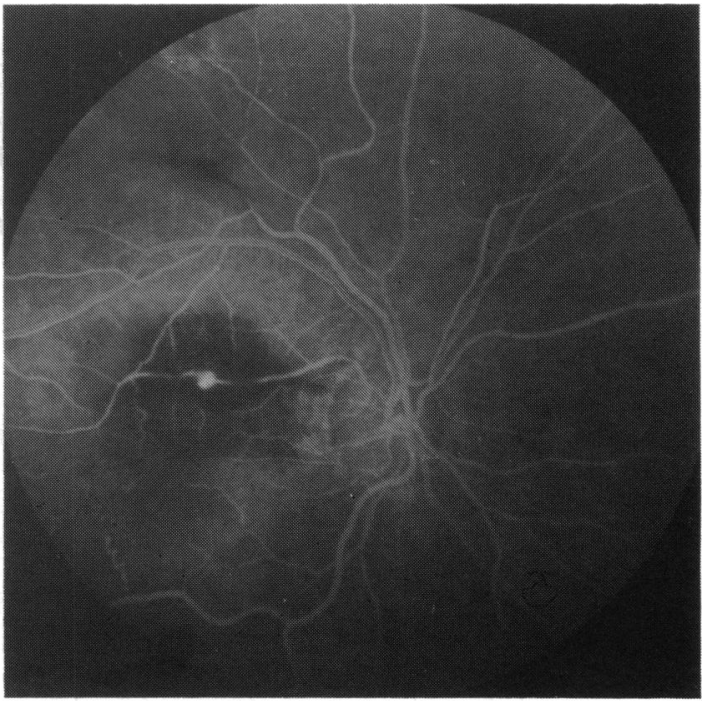

Fig. 4 Fluorescein angiography shows filling of the cilioretinal artery and the macroaneurysm. There is leakage from the macroaneurysm. 


\section{Discussion}

The natural history of retinal macroaneurysms is not completely known. Much has been said about the evolution of macroaneurysms after they acquire the typical round appearance and cause haemorrhages and exudates. However, much less is known about the changes in the arteries which precede the development of the macroaneurysm. It is probable that the arteries from which the macroaneurysms arise are seriously compromised, for the patients are generally older than $60^{245}$ and have hypertension and arteriosclerosis. ${ }^{1-5}$

Leishman ${ }^{8}$ reported that retinal arteries of older people with arteriosclerosis show thickening of the vessel wall and loss of contractile elements that are replaced by collagen tissue. Arteries modified in such a manner might dilate owing to pressure increase, thus producing macroaneurysms. Lewis et al. ${ }^{7}$ suggest that the production of macroaneurysms can follow focal damage of the vessel wall, especially at points where embolic plaques exist.

In the case described here we performed fundus photographs and fluorescein angiography before complete development of the macroaneurysm. In the tract where the macroaneurysm subsequently formed the artery showed slight enlargement, and its walls were hyalinised. Hyalinisation is a change that often accompanies arterial hypertension and develops in muscular arteries having a wall rich in collagen fibrils embedded in basal membrane-like material, as are the retinal arteries. ${ }^{9}$ Furthermore, in our case there were abnormalities of the endothelium with breakdown of the blood-retinal barrier: this was revealed by the presence of hard exudates around the tract of the artery involved. However, the increase in permeability was not considerable, as the fluorescein angiography did not show leakage.

The study of this case leads one to believe that, at least in certain patients, the development of a macroaneurysm passes through an early subclinical phase characterised by moderate changes in the artery and a late phase characterised by gross anatomical abnormalities of the vessel, with oedematous and haemorrhagic complications.

The evolution of the macroaneurysm from the time when it produces these complications has been described. ${ }^{261011}$ The macroaneurysm can show involutional changes: the walls may sclerose and the lumen become occluded or remain open. In addition, the artery from which the macroaneurysm originates may become occluded, narrow, or maintain a normal lumen. Intraretinal haemorrhages become reabsorbed without sequelae, but subretinal haemorrhages can produce disciform scars. Oedema is a more serious complication of macroaneurysms and can cause cystoid degeneration of the macula when it settles in this area. It is important to note that, irrespective of the location of the macroaneurysm, the oedema, as in other retinal vascular disease like Coats's disease and von Hippel capillary haemangioma, tends to be localised in the macula. ${ }^{12}$

Some authors have suggested not treating the macroaneurysms that cause haemorrhages, but photocoagulating those that cause macular oedema $^{.-7}$ If the macroaneurysm is small and far from the macula it can be directly photocoagulated; otherwise it is useful to perform photocoagulation all round the lesion. ${ }^{4513-15}$ Of course photocoagulation does not improve visual acuity if the oedema has already caused irreversible macular lesions.

As Robertson' stated, the location of the macroaneurysm is a retinal artery of the first three orders. Generally macroaneurysms are located in the superotemporal artery. ${ }^{46711}$ It is not clear, however, whether the higher frequency of macroaneurysms of the superotemporal artery is real or apparent, since a macroaneurysm at this site is apt to cause macular oedema and haemorrhages, with the consequent loss of vision that brings the patient to the ophthalmologist. Several macroaneurysms of the inferotemporal ${ }^{412}$ and nasal ${ }^{56}$ arteries have also been described. The case reported here, on the other hand, is one of the very rare cases of macroaneurysms of cilioretinal artery. ${ }^{\text {? }}$

The rarity of the location deserves some remarks. The cilioretinal artery is an artery of ciliary origin, while the retinal arteries arise from the central retinal artery. However, the haemodynamics of the ciliary arteries and retinal arteries are similar, in that their pressure are not much different. It is known that, in fluorescein angiography, the cilioretinal arteries fill together with, or often even before, the intraocular central retinal artery. The histological structure of the cilioretinal artery is the same as that of the first branches of the central retinal artery, consisting of a well developed muscular layer and a media and adventitia rich in collagen fibrils. It is understandable that the haemodynamic damage due to a hypertensive arterial disease could likewise involve the two arteries. Thus it is surprising that macroaneurysms of the cilioretinal artery are so much rarer than those of the central retinal artery despite an incidence of cilioretinal arteries in over $32 \%$ of the eyes in the general population. ${ }^{16}$

A possible explanation is that the greater length of the major branches of the intraocular central retinal artery as compared with the cilioretinal arteries could cause the former to be involved more frequently. Moreover, it is known that most macroaneurysms develop at arteriovenous crossings or where a vessel abruptly changes direction, ${ }^{17}$ points where the endo- 
thelium and the muscularis are more subject to haemodynamic stress. Thus arteries that present a tortuous course, arteriovenous crossings, and many subdivisions, like the temporal branches of the central retinal artery, may be more prone to develop macroaneurysms than arteries having a straighter course, no arteriovenous crossings, and few branches, like the macular cilioretinal arteries.

Since macroaneurysms are not very rare complications in patients with arterial hypertension, and since they frequently threaten the macula, periodic photographic follow-up that shows changes in the arterial wall, such as hyalinisation, and increased vessel permeability may be useful in patients at risk. Such controls would allow the possibility of immediate photocoagulative therapy to prevent the development of oedema and subsequent irreversible macular lesions.

\section{References}

1 Robertson DM. Macroaneurysms of the retinal arteries. Ophthalmology (Rochester) 1973; 77: 55-67.

2 Cleary PE, Kohner EM, Hamilton AM, Bird AC. Retinal macroaneurysms. Br J Ophthalmol 1975; 59: 355-61.

3 Nadel AJ, Gupta KK. Macroaneurysms of the retinal arteries. Arch Ophthalmol 1976; 94: 1092-6.
4 Dewachter A, De Laey JJ. Acquired retinal macroaneurysms. Bull Soc Belge Ophtalmol 1982; 201: 105-11.

5 Attali P, Sterkers M, Coscas G. Les macroanévrysmes artériels rétiniens. J Fr Ophtalmol 1984; 7: 697-710.

6 Palestine AG, Robertson DM, Goldstein BG. Macroaneurysms of the retinal arteries. Am J Ophthalmol 1982; 93: 164-71.

7 Lewis RA, Norton EWD, Gass JDM. Acquired arterial macroaneurysms of the retina. Br J Ophthalmol 1976; 60: 21-30.

8 Leishman $R$. The eye in general vascular disease, hypertension, and arteriosclerosis. Br J Ophthalmol 1957; 41: 641-701.

9 Sigelman J, Ozanics V. Retina. In: Duane TD, Jaeger EA, eds. Biochemical foundations of ophthalmology. Philadelphia: Harper and Row, 1982: i: 55.

10 Godel V, Blumenthal M, Regenbogen L. Arterial macroaneurysm of the retina. Ophthalmologica 1977; 175: 125-9.

11 François J. Acquired macroaneurysms of the retinal arteries. Int Ophthalmol 1979; 1: 153-61.

12 Asdourian GK, Goldberg MF, Jampol L, Rabb M. Retinal macroaneurysms. Arch Ophthalmol 1977; 95: 624-8.

13 Hudomel J, Imre G. Photocoagulation treatment of solitary aneurysm near the macula lutea. Acta Ophthalmol (Kbh) 1973; 51: $633-8$

14 Constantinides G, Hochart G. Macroanévrysme artériel rétinien. A propos d'un cas. Bull Soc Ophtalmol Fr 1981; 81: 579-81.

15 Van Nouhuys E, Deutman AF. Argon laser treatment of retinal macroaneurysms. Int Ophthalmol 1980; 2: 45-53.

16 Justice J, Lehmann RP. Cilioretinal arteries. Arch Ophthalmol 1976; 94: 1355-8.

Accepted for publication 29 July 1986. 